\title{
Serum Markers in the Clinical Management of Celiac Disease
}

\author{
Marlou Adriaanse Daniel A. Leffler \\ Division of Gastroenterology, Beth Israel Deaconess Medical Center, Boston, Mass., USA
}

\begin{abstract}
Key Words
Celiac disease - Serology - Tissue transglutaminase .

Deamidated gliadin peptide - Endomysial antibody .

Biomarker · Intestinal fatty acid-binding protein
\end{abstract}

\begin{abstract}
The advent of highly reliable noninvasive celiac diagnostic tests has transformed the field of celiac disease, from diagnosis, to evaluation of epidemiology, to clinical and translational research. Serologic tests in their modern forms are highly sensitive and specific for diagnosis, allowing for consideration of avoidance of diagnostic intestinal biopsy in some settings. On the other hand, as predictors of intestinal damage and for use in monitoring disease activity, currently available noninvasive tests have been disappointing. Serologic tests, while a measure of disease activity, do not correlate well with histology or symptomatology, and it is unclear if they predict long-term risk. Additionally, while the many clinically available tests have improved accessibility, they can have widely different cutoff levels and overall performance, making the comparison of levels in individual patients over time and across populations quite difficult. In the future, we can expect to see improvement in the currently available serologic tests including tissue transglutaminase and deamidated gliadin peptide with expansion of the dynamic range of the tests, and the celiac care community should push for a standardization of assays that would simplify research and patient care. Additionally, current serolog-
\end{abstract}

ic tests are measures of the adaptive immune response in celiac disease but do not directly measure intestinal inflammation. Promising work on intestinal fatty acid-binding protein and other assays which directly measure intestinal damage may complement traditional serologic tests and further improve our ability to noninvasively diagnose and monitor celiac disease. The coming years hold promise for the continuing evolution of serum-based tests in celiac disease with the possibility of substantial improvement of patient care and clinical research.

(c) 2015 S. Karger AG, Basel

\section{Introduction}

Celiac disease (CD) is a common immune-mediated enteropathy triggered by the intake of dietary gluten in wheat, barley and rye, in genetically susceptible individuals [1]. Gluten peptides in the intestine trigger T-helper 1 -mediated immune responses resulting in epithelial inflammation, an increased number of intraepithelial lymphocytes, intestinal villous atrophy, autoantibody production, and malabsorptive symptoms. Traditionally, the diagnosis of $\mathrm{CD}$ relies on visualizing the typical lesion via small intestinal biopsy. Early diagnosis and treatment of $\mathrm{CD}$ can prevent severe complications. A gluten-free diet (GFD) generally leads to improvement in intestinal mucosal damage, clinical symptoms and CD autoantibody titers.

\section{KARGER 125}

C 2015 S. Karger AG, Basel

$0257-2753 / 15 / 0332-0236 \$ 39.50 / 0$

E-Mail karger@karger.com

www.karger.com/ddi
Daniel A. Leffler

Division of Gastroenterology, Beth Israel Deaconess Medical Center

East/Dana 501, 330 Brookline Avenue

Boston, MA 02215-5400 (USA)

E-Mail dleffler@bidmc.harvard.edu 
The widespread availability of highly accurate serologic testing for CD has been a fundamental driver of advances in clinical care and research in this field [2]. The serologic tests for CD are more accurate than most other antibody-based tests for immune-based disorders. In the early 1960s, the gliadin component of wheat was found to be the pathologic trigger for CD. Autoantibodies (AGA) against this protein were subsequently the first to be used as a screening tool for the disease [3]. Since that time, serologic testing advanced from an adjunctive aid in diagnosis to an integral component of diagnosis, management and clinical research. Highly sensitive and specific tests including tissue transglutaminase (tTG), endomysial (EMA) and deamidated gliadin peptide (DGP) autoantibodies have been identified optimizing diagnostics and screening studies. Indeed, for all individuals in whom CD is being considered, serologic blood testing should be the initial step in evaluation [4-6]. With improvement of serologic antibody tests and highly accurate assays, we are potentially reaching the time where a diagnosis of $\mathrm{CD}$ can be made without intestinal biopsies, at least in select welldefined situations [5].

Despite these advances and the overall laudable test performance of EMA, tTG and DGP for CD diagnosis, current testing still is subject to a number of important limitations that are important for both clinicians and researchers to recognize. First, one of the most practical issues currently faced by clinicians is the diversity of available testing platforms, many of which have different cutoff levels, dynamic ranges and overall test performance. This issue, which has gone largely unaddressed, can be a major impediment to both patient care and research when values are not comparable between providers or between studies. In addition, it has been noted that the excellent test performance shown in validation studies of various serologic tests may not accurately reflect test performance in clinical practice and more diverse populations.

Furthermore, monitoring disease activity in treated $\mathrm{CD}$ patients remains a challenge. Although the CD antibody tests show a high accuracy for selecting patients needing a diagnostic biopsy, these tests do not seem to be reliable after diagnosis as the autoantibody titers do not correlate well with histological findings or symptoms in CD patients on a GFD. To optimize the noninvasive diagnostic algorithm and improve monitoring of disease activity, different tests for CD activity have been studied. Two promising markers are intestinal fatty acid-binding protein (I-FABP), a marker reflecting enterocyte damage, and citrulline (CIT), a marker for functional enterocyte mass, and will be discussed in this review.

Serum Markers in the Clinical Management of CD
Also, unlike histology, there are virtually no data on the impact of serologic test results of long-term prognosis. Fortunately, work is ongoing to address these limitations, and the coming years hold promise for the continuing evolution of serum-based tests in CD with the possibility of substantial improvement of patient care and clinical research. The aim of this review is to evaluate the currently available serum markers and their accuracy in clinical practice in patients with a suspicion of $C D$, and their use for monitoring disease activity in CD patients on a GFD. Next to the traditional (gliadin, reticulin, actin) and modern tests (tTG, EMA and DGP), two novel promising markers for disease activity (I-FABP and CIT) will be discussed.

\section{Traditional Autoantibody Tests: Anti-Gliadin, Anti-Reticulin and Anti-Actin}

\section{Anti-Reticulin and Anti-Actin}

Anti-reticulin antibodies (ARA) were first described as a possible predictive test for CD in the early 1970s [7]. While IgA antibodies to reticulin connective tissue did see some use, primarily in pediatric CD evaluation and appeared to have test performance equal to or better than AGA [8], ARA was largely supplanted first by AGA for technical reasons and later on by EMA and tTG which had markedly better test performance, as described below.

Around the same time as ARA were described, antiactin antibodies (AAA) were found to be present in a number of autoimmune and neoplastic conditions [9]. AAA has been evaluated on multiple occasions as a supplemental serologic test in CD. While AAA may have a higher predictive value for severe intestinal damage than other serologic tests, the relatively low sensitivity has made the clinical value of this test uncertain and AAA have never gained widespread use in $\operatorname{CD}[10,11]$.

\section{Anti-Gliadin}

About one decade later, in the 1980s, the native antigliadin antibody (AGA) test was developed for CD [12]. This test for both IgA and IgG antibodies was relatively easy to manufacture and perform (enzyme-linked immunosorbent assay, ELISA). It was a major step forward as they were the first tests to allow some degree of noninvasive risk stratification, especially important in what was, at that time, a primarily pediatric disease, and quickly became the standard serologic test.

There is marked heterogeneity in results of studies evaluating the sensitivity and specificity of AGA tests, as 
a results of assay variants and cutoffs provided, a common problem for all CD diagnostic tests [13-15]. The average sensitivity and specificity of the best tests for both $\operatorname{IgA}$ and IgG AGA is between 80 and $90 \%$, with lower specificities generally seen in adults compared with children $[2,13]$. In recent years, AGA-IgA tests have been replaced by more accurate serological assays, such as tTG, EMA and DGP.

One historic advantage of AGA had been the availability of a moderately accurate IgG-based test. While IgA is the major component of the gluten-autoantibody interaction in the small intestinal lumen, IgG represents a longer-term immune response. As selective IgA deficiency occurs in up to $2 \%$ of patients with CD, the need for a non-IgA-based test is apparent $[16,17]$. IgG AGA testing remained the standard diagnostic test for $\mathrm{CD}$ in individuals with selective IgA deficiency through the early 2000s. However, in the past few years, IgG-tTG and particularly IgG-DGP assays have been developed that are superior to IgG-AGA for this population [18-21].

Recently, AGA has been suggested as a marker of nonceliac gluten sensitivity (i.e. those with negative antibodies to tTG and/or EMA and normal intestinal histology) [22]. However, at this point, there are no clear data that support the use of AGA testing in any clinical setting where more modern tests are available.

\section{Newer Tests: EMA, tTG and DGP}

\section{Endomysium}

Introduction of the EMA test in the mid-1980s was a significant step forward from AGA testing. Although heterogenic, the sensitivity of EMA-IgA is generally reported to be above $90 \%$ with a specificity up to $100 \%[13,23,24]$. Because of the close to $100 \%$ specific association with CD, EMA testing has generally been used as a confirmatory test for tTG. However, approximately $10 \%$ of CD patients remain negative for EMA. The test has been suggested to be less sensitive in patients with milder small intestinal lesions (Marsh 3A) $[25,26]$ and among CD children under 2 years of age [27]. Nevertheless, different studies suggest that EMA appears in the serum before development of villous atrophy, making it a marker of early or 'potential' CD $[28,29]$. EMA is detected by an indirect immunofluorescence method (monkey esophagus or human umbilical cord tissue as antigen). A consequent disadvantage of this method is that the results are labor-intensive and operator-dependent, increasing the costs and diminishing the objectivity of the test [2].

\section{Tissue Transglutaminase}

The identification of tTG as autoantigen in the late 1990s enabled the development of an easy to perform ELISA-based test with recombinant tTG. tTG is responsible for the deamidation of gluten-derived gliadin peptides, resulting in DGP with greatly enhanced immunoreactivity $[30,31]$. Gluten in this altered confirmation binds with high affinity to HLA DQ2 or HLA DQ8 molecules on antigen-presenting cells which present the deamidated gluten and bound tTG to helper T cells triggering production of antibodies to tTG and DGP [32-35]. Enteropathy is caused by cell-mediated cytotoxicity triggered also by the binding of deamidated gluten to HLA molecules on antigen-presenting cells but resulting in CD8-mediated inflammation with infiltration of lymphocytes into the mucosa along with villous destruction. (The pathogenic significance of the autoantibody response in CD patients remains unclear; however, celiac antibodies have been postulated to increase intestinal permeability, activate monocytes, disturb angiogenesis, inhibit epithelial cell differentiation or induce cell proliferation.) Since they were described, tTG quickly became the standard test used in $\mathrm{CD}$ diagnosis, as this method is more reproducible and objective compared with EMA. The best tests for tTG IgA have shown both sensitivity and specificity of $95 \%$ and above $[2,13,14,36]$. The performance of commercial ELISA assays for $\mathrm{tTG}$ however may vary depending on the quality of the antigen, and discrepancies between different kits are also present in the cutoff values. The concordance rate of tTG and EMA test is very high, but based on its high sensitivity, lower costs and ease of interpretation, tTG is suggested as the first screening test $[37,38]$. Nevertheless, in most studies, the specificity of EMA tests is higher, suggesting that this test can be performed as confirmatory in tTG-positive individuals $[38,39]$. Furthermore, as discussed above, the accuracy of all serologic tests depends on disease prevalence and may be lower in clinical settings compared with highly enriched validation cohorts.

\section{Deamidated Gliadin Peptides}

Most recently, drawing off the known pathophysiology of $\mathrm{CD}$, antibodies directed to DGP have been introduced into the spectrum of serologic tests for CD. DGP reflects the $\mathrm{CD} B$-cell epitopes more specific than the native nonamidated peptides targeted by the traditional AGA [20], and consequently has a higher sensitivity and specificity. Although DGP-IgA was shown to be nearly as sensitive and specific as tTG-IgA [40,41], recent studies revealed that $\mathrm{tTG}$ performs significantly better $[36,42]$. The sensitivity and specificity for DGP-IgA have gener- 
ally been shown to be around 85 and $95 \%$, respectively [2, $36,43]$. Nevertheless, while IgG-based tTG tests in IgAdeficient patients have disappointing sensitivity, DGPIgG tests display high specificity for CD $(99 \%$, similar to EMA) [20, 40, 42-44]. DGP-IgG allows for the identification of all CD cases in IgA-deficient patients with a high sensitivity in young children, proposing a new protocol for CD that uses only two tests.

The revolution in antibody tests has substantially improved the diagnostic algorithm for CD. Further improvement can be expected by rapid point of care tests that can be performed at the outpatient clinic, instead of requiring trained personnel and centralized laboratories. Point of care tTG-IgA tests have been developed, but reports on their accuracy are contradictory, and these tests need further exploration before clinical implementation $[39,45]$.

Despite the major improvements in serologic testing, no antibody test shows an absolute sensitivity and specificity for $\mathrm{CD}$. In the majority of patients, the diagnosis of $\mathrm{CD}$ still relies on the demonstration of the characteristic small intestinal changes by duodenal biopsy. The best serologic approach for case finding might be the combined detection of a panel of antibodies. Recently, the European Society for Pediatric Gastroenterology Hepatology and Nutrition introduced a revised guideline for CD in children based on the optimization of antibody assays. According to this guideline, children can be diagnosed with $\mathrm{CD}$ without a duodenal biopsy when presenting with symptoms, a strongly elevated tTG-IgA ( $>10$ cutoff value), confirmed by a positive EMA-IgA test on a separate occasion, and positive genetic testing (HLA-DQ2 and/or HLA-DQ8) [5]. Such a strategy seems premature for CD in adults, and a substantial proportion of children with suspicion of CD do not fulfill all four diagnostic criteria. Thus, currently a diagnostic biopsy remains necessary for most patients.

Other serum markers are warranted to prevent the diagnostic biopsies in all children and in adult patients. Research has been underway for many years for a noninvasive test that cannot only screen individuals at risk for $\mathrm{CD}$, but also correlate with the severity of the intestinal lesions and monitor the histological response to a GFD. Potential markers are discussed below.

\section{Experimental Tests: I-FABP and CIT}

\section{Citrulline}

CIT is a nonprotein amino acid, and is a metabolic intermediate in the urea cycle. CIT is almost exclusively present in the enterocytes of the small intestine [46-48].
Consequently, circulating CIT levels have been proposed to be a potential marker of small intestinal function and mass. Recently, CIT levels have been shown to be a reliable marker of small intestinal damage in other disease associated with villous atrophy and enterocyte alterations, including HIV and tropical enteropathy $[49,50]$.

Publications on the value of CIT testing in CD are conflicting. A few studies showed that CIT levels are reduced in children and adults with untreated CD compared with healthy controls [51-53]. Most of these studies showed an inverse correlation between fasting plasma CIT level and the extent of villous atrophy $[51,52]$. However, significant overlap was present between CD patients and controls [52, 53]. Furthermore, others found that fasting plasma CIT levels were unable to reflect a decreased intestinal energy absorption capacity in patients with enterocyte damage [54]. This may be due to a milder degree of intestinal disease [54], suggesting that significant intestinal damage must be present for CIT levels to decrease.

Initiation of a GFD in studies with decreased CIT levels at diagnosis led to an increase in CIT levels [51-53], depending on the histological responsiveness [51], and has been shown to normalize CIT levels to the levels of controls after more than 2 years of GFD [52].

As an alternative to fasting CIT levels, a CIT generation test (CGT) has been provided to assess enterocyte function [55]. Loading the enterocyte with orally administered glutamine, the natural precursor of CIT, may increase CIT output, reflecting the functional capacity of the enterocyte. This study revealed that the generation of CIT was delayed in patients with CD. This test might offer a new possibility to assess the functional capacity of enterocytes in CD. For now however, the clinical utility of CIT measurement is questionable in $\mathrm{CD}$ and needs further evaluation.

\section{Intestinal Fatty Acid-Binding Protein}

Measurement of circulating endogenous enterocyte proteins has been shown to be useful to estimate enterocyte damage. Fatty acid-binding protein comprises a class of low-molecular-weight (14-15 kDa) cytosolic proteins found in high concentrations in tissues involved in the uptake and consumption of fatty acids [56]. Three types of FABP are present in the gut: intestinal FABP (I-FABP), liver FABP (L-FABP) and ileal bile acid-binding protein (I-BABP). I-FABP is executively present in the gut, as is I-BABP which is exclusively present in the ileum, while L-FABP is also present in the liver and kidneys. I-FABP is primarily limited to mature enterocytes of the small intestine, with a maximal expression in the jejunum, while 
the expression in the colon is low [57]. Since I-FABPs are small water-soluble proteins, they are easily released into the circulation upon enterocyte damage and rapidly cleared though the kidneys (half-life of $11 \mathrm{~min}$ ). I-FABP levels can be measured sensitively using an ELISA. It circulates in low amounts in the bloodstream of healthy individuals, likely reflecting the physiological turnover rate of enterocytes. Outside of CD, elevated circulating FABP levels have been reported in patients with intestinal ischemia, necrotizing enterocolitis and the early phase of sepsis [58-60].

Recent studies have revealed that serum I-FABP may be a sensitive marker for enterocyte damage in $\mathrm{CD}$. These studies showed that I-FABP levels are significantly elevated in untreated $\mathrm{CD}$ adults and children at diagnosis as compared with control subjects $[57,61,62]$. Interestingly, serum I-FABP levels correlated with the severity of the histological lesions. These studies showed a sensitivity of 80 and $82 \%$, and a specificity of 87 and $90 \%$ in adults and children, respectively, for $\mathrm{CD}$, suggesting that serum IFABP might be a useful additional marker in the diagnostic algorithm of CD $[61,62]$. Patients with positive CDspecific markers and an elevated serum I-FABP level seem to suffer from $C D$, suggesting that a diagnostic biopsy can be omitted in these patients. Nevertheless, in patients with elevated $\mathrm{CD}$, autoantibody titers and a normal serum I-FABP level, a duodenal biopsy would remain necessary.

Serum I-FABP levels may also be useful for monitoring disease activity after diagnosis. After introduction of the GFD, serum I-FABP levels decrease relatively fast. This is especially useful to confirm a diagnosis made without duodenal biopsy. In children, serum I-FABP levels have been shown to generally decrease and normalize within 6 months after initiation of gluten elimination [61]. This is faster than the decrease in tTG levels. However, in adult patients, levels appeared elevated compared with controls, even after up to 3-4 years of apparent compliance with the GFD [62]. This is in line with studies on histological healing in patients on a GFD, generally showing slow and incomplete mucosal healing in adult $\mathrm{CD}$ patients, while recovery in children seems to be faster and more complete $[63,64]$. As postulated, a correlation has been found between serum I-FABP levels and the severity of the histological lesions in CD patients on a GFD [62]. Furthermore, a pilot study showed that I-FABP may be used as an early responsive marker during a gluten challenge procedure [unpubl. data]. Large international studies are underway to validate this marker for use in diagnosis, monitoring and research trials.

\section{Limitations and Areas for Future Study}

$\mathrm{CD}$ is a significant disorder with limited treatment adherence $[65,66]$ and adverse effects on quality of life $[65$, $67,68]$ and nutritional [69] and metabolic indices [70, 71]. It is also associated with increased risk of malignancy and mortality [72-74]. For this reason, all major societies recommend routine follow-up of patients with CD [4-6, 75]. The goal of routine monitoring is to identify and treat coexisting disorders and manage active CD.

Thus, reliable but also easily applicable markers are needed. The modalities commonly used for CD monitoring are serologic tests and less often intestinal biopsy. While both tests are clearly supported as part of CD diagnosis, their use in monitoring is much less clear. Both small intestinal mucosal inflammation and serologic tests are reflections of different aspects of the adaptive immune response triggered by gluten in $\mathrm{CD}$. When $\mathrm{CD}$ is highly active, as at diagnosis before the GFD is initiated, highly elevated serologic titers are correlated with intestinal damage, as both arms of adaptive immunity are fully activated $[2,76]$. With initiation of the GFD however, this relationship becomes unstable. Data show that EMA, tTG and DGP titers should fall significantly in the first months after initiation of GFD and be normal or close to normal one year after diagnosis [77-79]. It is clear from gluten challenge studies that increases in antibody titers reflect increased disease activity and increased intestinal inflammation. However, elevation of CD-specific serology is delayed significantly from episodes of gluten exposure [80], and $\mathrm{CD}$ antibodies are poor predictors of dietary adherence with low levels of cross contamination or inadvertent gluten [81, 82]. CD serology lacks both sensitivity and specificity for ongoing enteropathy on a long-term GFD [83]. Furthermore, there are no studies that evaluate the relationship between normalization of serologic tests and risk of long-term complications. Although more research is necessary, promising experimental markers for enterocyte damage, such as I-FABP and CIT, may be more accurate for monitoring disease activity in $\mathrm{CD}$ patients on a GFD.

\section{Conclusion}

Serum markers for CD play an important role in CD management. In recent decades, the evolvement from detection of the disease trigger to several antibody tests has greatly improved the diagnostic algorithm, epidemiology and research, and will continue to do so. 
In the upcoming years, further improvement and expansion of existing and new serological tests can be expected, with wider dynamic ranges, improved responsiveness and possibly higher sensitivity and specificity. Combination of different noninvasive markers may lead to diagnostic algorithms with greatly reduced need for diagnostic duodenal biopsies. Novel markers measuring direct mucosal damage, like I-FABP and CIT, instead of reflecting the immune response, may complement traditional serologic tests. These markers may also be more accurate predictors for monitoring disease activity after diagnosis, as the currently used antibody tests do not correlate well with histology or symptomatology. Once these novel tests are validated and clinically available, guidelines for $\mathrm{CD}$ diagnosis and monitoring may look very different from those available today.

At the same time, use of different antibody tests and cutoffs make comparison in individual patients over time and across populations difficult in both clinical and research situations. The range of competing tests is likely only to grow in the coming years. A centralized effort to perform an open and robust comparison across testing platforms paired with detailed clinical and histologic data is feasible and could be completed with modest resources. These data would also be essential to the next major question of whether serologic titers, either at diagnosis or in follow-up, are of any prognostic significance in diagnosed patients. A number of studies have provided important data on the relationship between histology and long-term outcomes in CD. Clearly, the fact that we have only had serologic tests for a few decades has limited the conduct of these studies. However, sufficient time has elapsed to begin to make these studies possible.

\section{Disclosure Statement}

Daniel Leffler has been a consultant for and/or received research support from the following: NIH, Shire Pharmaceuticals, Inova Diagnostics, Coranado Biosciences, Alba Therapeutics, Alvine Pharmaceuticals, Glenwood Pharmaceuticals and Ironwood Pharmaceuticals.

\section{References}

$\checkmark 1$ Green PH, Cellier C: Celiac disease. N Engl J Med 2007;357:1731-1743.

-2 Leffler DA, Schuppan D: Update on serologic testing in celiac disease. Am J Gastroenterol 2010;105:2520-2524.

3 Berger E, Buergin-Wolff A, Freudenberg E: Diagnostic value of the demonstration of gliadin antibodies in celiac disease (in German). Klin Wochenschr 1964;42:788-790.

4 Rubio-Tapia A, Hill ID, Kelly CP, et al: ACG clinical guidelines: diagnosis and management of celiac disease. Am J Gastroenterol 2013;108:656-676; quiz 677.

5 Husby S, Koletzko S, Korponay-Szabo IR, et al: European Society for Pediatric Gastroenterology, Hepatology, and Nutrition guidelines for the diagnosis of coeliac disease. J Pediatr Gastroenterol Nutr 2012;54:136-160.

-6 Bai JC, Fried M, Corazza GR, et al: World Gastroenterology Organisation global guidelines on celiac disease. J Clin Gastroenterol 2013;47:121-126.

7 Seah PP, Fry L, Rossiter MA, et al: Anti-reticulin antibodies in childhood coeliac disease. Lancet 1971;2:681-682.

-8 Lerner A, Kumar V, Iancu TC: Immunological diagnosis of childhood coeliac disease: comparison between antigliadin, antireticulin and antiendomysial antibodies. Clin Exp Immunol 1994;95:78-82.

9 Trenchev P, Holborow EJ: The specificity of anti-actin serum. Immunology 1976;31:509517.

Serum Markers in the Clinical

Management of CD
10 Fabbro E, Rubert L, Quaglia S, et al: Uselessness of anti-actin antibody in celiac disease screening. Clin Chim Acta 2008;390:134-137.

11 Granito A, Muratori P, Cassani F, et al: Antiactin IgA antibodies in severe coeliac disease. Clin Exp Immunol 2004;137:386-392.

12 O'Farrelly C, Kelly J, Hekkens W, et al: Alpha gliadin antibody levels: a serological test for coeliac disease. BMJ (Clin Res Ed) 1983;286: 2007-2010.

13 Hill ID: What are the sensitivity and specificity of serologic tests for celiac disease? Do sensitivity and specificity vary in different populations? Gastroenterology 2005;128:S25-S32.

14 Rostom A, Dube C, Cranney A, et al: The diagnostic accuracy of serologic tests for celiac disease: a systematic review. Gastroenterology 2005;128:S38-S46.

15 Naiyer AJ, Hernandez L, Ciaccio EJ, et al: Comparison of commercially available serologic kits for the detection of celiac disease. J Clin Gastroenterol 2009;43:225-232.

16 Cataldo F, Marino V, Bottaro G, et al: Celiac disease and selective immunoglobulin A deficiency. J Pediatr 1997;131:306-308.

17 Chow MA, Lebwohl B, Reilly NR, et al: Immunoglobulin A deficiency in celiac disease. J Clin Gastroenterol 2012;46:850-854.

18 Dahlbom I, Olsson M, Forooz NK, et al: Immunoglobulin $\mathrm{G}(\mathrm{IgG})$ anti-tissue transglutaminase antibodies used as markers for IgAdeficient celiac disease patients. Clin Diagn Lab Immunol 2005;12:254-258.
19 Cataldo F, Lio D, Marino V, et al: IgG(1) antiendomysium and IgG antitissue transglutaminase (anti-tTG) antibodies in coeliac patients with selective IgA deficiency. Working Groups on Celiac Disease of SIGEP and Club del Tenue. Gut 2000;47:366-369.

20 Prince HE: Evaluation of the INOVA diagnostics enzyme-linked immunosorbent assay kits for measuring serum immunoglobulin $G$ (IgG) and IgA to deamidated gliadin peptides. Clin Vaccine Immunol 2006;13:150-151.

21 Lenhardt A, Plebani A, Marchetti F, et al: Role of human-tissue transglutaminase $\operatorname{IgG}$ and anti-gliadin IgG antibodies in the diagnosis of coeliac disease in patients with selective immunoglobulin A deficiency. Dig Liver Dis 2004;36:730-734.

22 Verdu EF, Armstrong D, Murray JA: Between celiac disease and irritable bowel syndrome: the 'no man's land' of gluten sensitivity. Am J Gastroenterol 2009;104:1587-1594.

23 Collin P, Kaukinen K, Vogelsang H, et al: Antiendomysial and antihuman recombinant tissue transglutaminase antibodies in the diagnosis of coeliac disease: a biopsy-proven European multicentre study. Eur J Gastroenterol Hepatol 2005;17:85-91.

24 Volta U, Fabbri A, Parisi C, et al: Old and new serological tests for celiac disease screening. Expert Rev Gastroenterol Hepatol 2010;4: 31-35. 
25 Rostami K, Kerckhaert J, Tiemessen R, et al: Sensitivity of antiendomysium and antigliadin antibodies in untreated celiac disease: disappointing in clinical practice. Am J Gastroenterol 1999;94:888-894.

26 Abrams JA, Diamond B, Rotterdam H, et al: Seronegative celiac disease: increased prevalence with lesser degrees of villous atrophy. Dig Dis Sci 2004;49:546-550.

27 Maglio M, Tosco A, Paparo F, et al: Serum and intestinal celiac disease-associated antibodies in children with celiac disease younger than 2 years of age. J Pediatr Gastroenterol Nutr 2010;50:43-48.

28 Maki M, Holm K, Lipsanen V, et al: Serological markers and HLA genes among healthy first-degree relatives of patients with coeliac disease. Lancet 1991;338:1350-1353.

29 Kurppa K, Collin P, Viljamaa M, et al: Diagnosing mild enteropathy celiac disease: a randomized, controlled clinical study. Gastroenterology 2009; 136:816-823.

- 30 Molberg O, McAdam SN, Korner R, et al: Tissue transglutaminase selectively modifies gliadin peptides that are recognized by gut-derived T cells in celiac disease. Nat Med 1998; 4:713-717.

31 Dieterich W, Ehnis T, Bauer M, et al: Identification of tissue transglutaminase as the autoantigen of celiac disease. Nat Med 1997;3: 797-801.

32 Abadie V, Sollid LM, Barreiro LB, et al: Integration of genetic and immunological insights into a model of celiac disease pathogenesis. Annu Rev Immunol 2011;29:493-525.

-33 Jabri B, Sollid LM: Mechanisms of disease: immunopathogenesis of celiac disease. Nat Clin Pract Gastroenterol Hepatol 2006;3: 516-525.

-34 Schuppan D, Junker Y, Barisani D: Celiac disease: from pathogenesis to novel therapies. Gastroenterology 2009;137:1912-1933.

35 Sollid LM, Jabri B: Celiac disease and transglutaminase 2: a model for posttranslational modification of antigens and HLA association in the pathogenesis of autoimmune disorders. Curr Opin Immunol 2011;23:732738.

-36 Lewis NR, Scott BB: Meta-analysis: deamidated gliadin peptide antibody and tissue transglutaminase antibody compared as screening tests for coeliac disease. Aliment Pharmacol Ther 2010;31:73-81.

37 Sardy M, Karpati S, Peterfy F, et al: Comparison of a tissue transglutaminase ELISA with the endomysium antibody test in the diagnosis of gluten-sensitive enteropathy. Z Gastroenterol 2000;38:357-364.

38 Tonutti E, Visentini D, Bizzaro N, et al: The role of antitissue transglutaminase assay for the diagnosis and monitoring of coeliac disease: a French-Italian multicentre study. J Clin Pathol 2003;56:389-393.
39 Raivio T, Korponay-Szabo IR, Paajanen T, et al: Comparison of a novel whole blood transglutaminase-based ELISA with a whole blood rapid antibody test and established conventional serological celiac disease assays. J Pediatr Gastroenterol Nutr 2008;47:562-567.

40 Sugai E, Vazquez H, Nachman F, et al: Accuracy of testing for antibodies to synthetic gliadin-related peptides in celiac disease. Clin Gastroenterol Hepatol 2006;4:1112-1117.

41 Volta U, Granito A, Fiorini E, et al: Usefulness of antibodies to deamidated gliadin peptides in celiac disease diagnosis and follow-up. Dig Dis Sci 2008; 53:1582-1588.

42 Volta U, Granito A, Parisi C, et al: Deamidated gliadin peptide antibodies as a routine test for celiac disease: a prospective analysis. J Clin Gastroenterol 2010;44:186-190.

43 Volta U, Villanacci V: Celiac disease: diagnostic criteria in progress. Cell Mol Immunol 2011;8:96-102.

44 Villalta D, Tonutti E, Prause C, et al: IgG antibodies against deamidated gliadin peptides for diagnosis of celiac disease in patients with IgA deficiency. Clin Chem 2010;56:464-468.

45 Korponay-Szabo IR, Szabados K, Pusztai J, et al: Population screening for coeliac disease in primary care by district nurses using a rapid antibody test: diagnostic accuracy and feasibility study. BMJ 2007;335:1244-1247.

-46 Windmueller HG, Spaeth AE: Source and fate of circulating citrulline. Am J Physiol 1981; 241:E473-E480.

-47 Wakabayashi Y, Henslee JG, Jones ME: Pyrroline-5-carboxylate synthesis from glutamate by rat intestinal mucosa. Subcellular localization and temperature stability. J Biol Chem 1983;258:3873-3882.

-48 Wakabayashi Y, Yamada E, Hasegawa T, et al: Enzymological evidence for the indispensability of small intestine in the synthesis of arginine from glutamate. I. Pyrroline-5-carboxylate synthase. Arch Biochem Biophys 1991; 291:1-8.

49 Crenn P, De Truchis P, Neveux N, et al: Plasma citrulline is a biomarker of enterocyte mass and an indicator of parenteral nutrition in HIV-infected patients. Am J Clin Nutr 2009;90:587-594.

50 Papadia C, Kelly P, Caini S, et al: Plasma citrulline as a quantitative biomarker of HIVassociated villous atrophy in a tropical enteropathy population. Clin Nutr 2010;29:795800.

51 Crenn P, Vahedi K, Lavergne-Slove A, et al: Plasma citrulline: a marker of enterocyte mass in villous atrophy-associated small bowel disease. Gastroenterology 2003;124:1210-1219.

52 Ioannou HP, Fotoulaki M, Pavlitou A, et al: Plasma citrulline levels in paediatric patients with celiac disease and the effect of a glutenfree diet. Eur J Gastroenterol Hepatol 2011; 23:245-249.

53 Miceli E, Poggi N, Missanelli A, et al: Is serum citrulline measurement clinically useful in coeliac disease? Intern Emerg Med 2008;3: 233-236.
54 Peters JH, Wierdsma NJ, Teerlink T, et al: Poor diagnostic accuracy of a single fasting plasma citrulline concentration to assess intestinal energy absorption capacity. Am J Gastroenterol 2007;102:2814-2819.

55 Peters JH, Wierdsma NJ, Teerlink T, et al: The citrulline generation test: proposal for a new enterocyte function test. Aliment Pharmacol Ther 2008;27:1300-1310.

56 Pelsers MM, Hermens WT, Glatz JF: Fatty acid-binding proteins as plasma markers of tissue injury. Clin Chim Acta 2005;352:15-35.

57 Derikx JP, Vreugdenhil AC, Van den Neucker AM, et al: A pilot study on the noninvasive evaluation of intestinal damage in celiac disease using I-FABP and L-FABP. J Clin Gastroenterol 2009;43:727-733.

58 Derikx JP, Poeze M, van Bijnen AA, et al: Evidence for intestinal and liver epithelial cell injury in the early phase of sepsis. Shock 2007; 28:544-548.

59 Derikx JP, Evennett NJ, Degraeuwe PL, et al: Urine based detection of intestinal mucosal cell damage in neonates with suspected necrotising enterocolitis. Gut 2007;56:1473-1475.

60 Schellekens DH, Grootjans J, Dello SA, et al: Plasma intestinal fatty acid-binding protein levels correlate with morphologic epithelial intestinal damage in a human translational ischemia-reperfusion model. J Clin Gastroenterol 2014;48:253-260.

61 Vreugdenhil AC, Wolters VM, Adriaanse MP, et al: Additional value of serum I-FABP levels for evaluating celiac disease activity in children. Scand J Gastroenterol 2011;46: 1435-1441.

62 Adriaanse MP, Tack GJ, Passos VL, et al: Serum I-FABP as marker for enterocyte damage in coeliac disease and its relation to villous atrophy and circulating autoantibodies. Aliment Pharmacol Ther 2013;37:482-490.

63 Wahab PJ, Meijer JW, Mulder CJ: Histologic follow-up of people with celiac disease on a gluten-free diet: slow and incomplete recovery. Am J Clin Pathol 2002;118:459-463.

64 Bardella MT, Velio P, Cesana BM, et al: Coeliac disease: a histological follow-up study. Histopathology 2007;50:465-471.

65 Lee AR, Ng DL, Diamond B, et al: Living with coeliac disease: survey results from the USA. J Hum Nutr Diet 2012;25:233-238.

66 Leffler DA, Edwards-George J, Dennis M, et al: Factors that influence adherence to a gluten-free diet in adults with celiac disease. Dig Dis Sci 2008;53:1573-1581.

67 Mahadev S, Simpson S, Lebwohl B, et al: Is dietitian use associated with celiac disease outcomes? Nutrients 2013;5:1585-1594.

68 Barratt SM, Leeds JS, Sanders DS: Quality of life in coeliac disease is determined by perceived degree of difficulty adhering to a gluten-free diet, not the level of dietary adherence ultimately achieved. J Gastrointest Liver Dis 2011;20:241-245. 
69 Theethira TG, Dennis M, Leffler DA: Nutritional consequences of celiac disease and the gluten-free diet. Expert Rev Gastroenterol Hepatol 2014;8:123-129.

$>70$ Kabbani TA, Goldberg A, Kelly CP, et al: Body mass index and the risk of obesity in coeliac disease treated with the gluten-free diet. Aliment Pharmacol Ther 2012;35:723729.

71 Abid N, McGlone O, Cardwell C, et al: Clini$\mathrm{cal}$ and metabolic effects of gluten free diet in children with type 1 diabetes and coeliac disease. Pediatr Diabetes 2011;12:322-325.

72 Lebwohl B, Granath F, Ekbom A, et al: Mucosal healing and mortality in coeliac disease. Aliment Pharmacol Ther 2013;37:332-339.

73 Biagi F, Corazza GR: Mortality in celiac disease. Nat Rev Gastroenterol Hepatol 2010;7: 158-162.

74 Rubio-Tapia A, Rahim MW, See JA, et al: Mucosal recovery and mortality in adults with celiac disease after treatment with a gluten-free diet. Am J Gastroenterol 2010;105:14121420 .
75 AGA Institute: AGA Institute medical position statement on the diagnosis and management of celiac disease. Gastroenterology 2006;131:1977-1980.

76 Tursi A, Brandimarte G, Giorgetti GM: Prevalence of antitissue transglutaminase antibodies in different degrees of intestinal damage in celiac disease. J Clin Gastroenterol 2003;36:219-221.

77 Midhagen G, Aberg AK, Olcen P, et al: Antibody levels in adult patients with coeliac disease during gluten-free diet: a rapid initial decrease of clinical importance. J Intern Med 2004;256:519-524.

78 Dickey W, Hughes DF, McMillan SA: Disappearance of endomysial antibodies in treated celiac disease does not indicate histological recovery. Am J Gastroenterol 2000;95:712714.
79 Dipper CR, Maitra S, Thomas R, et al: Antitissue transglutaminase antibodies in the follow-up of adult coeliac disease. Aliment Pharmacol Ther 2009;30:236-244.

80 Leffler D, Schuppan D, Pallav K, et al: Kinetics of the histological, serological and symptomatic responses to gluten challenge in adults with coeliac disease. Gut 2013;62:996-1004.

-81 Leffler DA, Edwards George JB, Dennis M, et al: A prospective comparative study of five measures of gluten-free diet adherence in adults with coeliac disease. Aliment Pharmacol Ther 2007;26:1227-1235.

82 Vahedi K, Mascart F, Mary JY, et al: Reliability of antitransglutaminase antibodies as predictors of gluten-free diet compliance in adult celiac disease. Am J Gastroenterol 2003;98: 1079-1087.

83 Tursi A, Brandimarte G, Giorgetti GM: Lack of usefulness of anti-transglutaminase antibodies in assessing histologic recovery after gluten-free diet in celiac disease. J Clin Gastroenterol 2003;37:387-391. 